\title{
EL TRABAJO DEL PROFESIONAL DE ENFERMERÍA: REVISIÓN DE LA LITERATURA
}

\section{THE WORK OF PROFESSIONAL NURSING: A LITERATURE REVIEW}

\author{
Fredy Seguel Palma* \\ Sandra ValenZuela Suazo ${ }^{* *}$ \\ Olivia Sanhueza Alvarado ${ }^{* *}$
}

\begin{abstract}
RESUMEN
Objetivo: El objetivo de esta revisión de la literatura es analizar las características del "trabajo" en enfermería, inmersa en el mundo globalizado de la época contemporánea, de acuerdo a los resultados obtenidos en diferentes publicaciones que se refieren al tema. Material y método: Revisión bibliográfica desde los años 2000 al 2010 en bases de datos informatizadas: Web of Science, MEDLINE, PubMed SciELO, LILACS, BIREME y CUIDEN, de artículos a texto completo que presentaron en el título los siguientes descriptores: "trabajo", "enfermería", "salud laboral" o "rol de la enfermera". Resultados: 39 estudios cumplieron con los criterios de inclusión. Se distinguen las siguientes temáticas abordadas en las investigaciones: "actividades realizadas por los enfermeros", "condiciones de trabajo en las cuales se desempeñan", "el género como condición característica", "consecuencias producto del trabajo excesivo" y "satisfacción laboral". Conclusión: Las funciones y actividades del profesional de enfermería se distribuyen en diferentes ámbitos, con múltiples satisfacciones, pero en muchas ocasiones se realizan en condiciones deficientes, con alta demanda y estrés, pero con motivación y compromiso.
\end{abstract}

Palabras clave: Trabajo, enfermería, salud laboral, condiciones de trabajo.

\begin{abstract}
Objective: The aim of this review is to analyze the characteristics of "work" immersed in the globalized world of contemporary nursing time, according to the results obtained in different publications that talk about it. Methods: Literature review from 2000 to 2010 years on computer databases: Web of Science, MEDLINE, PubMed, SciELO, LILACS, BIREME and CUIDEN, full-text articles that appeared in the title the following descriptors: "Work", "nursing", "occupational health" or "role of the nurse". We obtained 39 studies that met inclusion criteria. Results: The following themes can distinguish in the different research: "activities performed by nurses", "working conditions", "Gender as a characteristic condition", "product consequences of overwork" and "job satisfaction". Conclusion: The functions and activities of the nurse are distributed in different areas, with multiple satisfactions, but often are held in poor conditions, with high demand and stress, but with motivation and commitment.
\end{abstract}

Key words: Work, nursing, occupational health, working conditions.

Fecha recepción: 09/04/14 Fecha aceptación: 06/04/15

\footnotetext{
*Enfermero, Magíster en Salud Pública, Doctor en Enfermería, Universidad de Concepción. Docente Instituto de Enfermería, Facultad de Medicina, Universidad Austral de Chile. Valdivia-Chile. Email: fredyseguel@uach.cl

${ }^{*}$ Enfermera, Doctora en Enfermería, Docente Facultad de Enfermería. Universidad de Concepción, Concepción-Chile.

Email: svalenzu@udec.cl

${ }^{* * *}$ Enfermera, Doctora en Enfermería, Docente Facultad de Enfermería. Universidad de Concepción, Concepción-Chile. Email: osanhue@udec.cl
} 


\section{INTRODUCCIÓN}

El trabajo está influenciado por la competencia que ha establecido el mercado entre las diferentes empresas, provocando en muchas ocasiones desgaste en los trabajadores en torno al mantenimiento de la actividad productiva, con bajas remuneraciones, pero con exceso de trabajo. El estándar de vida que busca la persona para sí o para su familia en torno al constructo consumista, da paso al surgimiento de la extensión horaria, la multiplicidad de empleos y los empleos informales (1).

La actividad laboral como relación contractual se transforma en un modo de inserción social subjetivamente motivadora, si la persona está calificada y a gusto, pero destructivo cuando las actividades son monótonas o desprovistas de sentido (2).

Las/los enfermeras/os se desempeñan (26) tanto en áreas intrahospitalarias como extrahospitalarias o comunitarias y, dentro de ellas, en los ámbitos: asistencial, gestión, educación e investigación. El cuidado de la persona con necesidades es su principal prioridad, involucrándose no solo con el paciente, sino que además con la familia y comunidad, asociando tiempo, energía y sentimientos. Es catalogada como la cuarta profesión más estresante asociada, también, al bajo reconocimiento social (7).

La/el enfermera/o está en constante presión de trabajo, enfrentada al dolor, sufrimiento, desesperanza, muerte, desconsuelo, impotencia y angustia; además, la falta de trabajadores que puedan abarcar a cabalidad los requerimientos de la población y de las instituciones empleadoras hace que doblen su jornada laboral $(8,9)$. Si sufre daño, por el cansancio emocional que traen sus labores y el desequilibrio de las interrelaciones con colegas, la organización, los pacientes y la carga de trabajo, ello provoca deterioro en la calidad del cuidado (10).

Realiza su labor en un ambiente donde son preponderantes los riesgos biológicos, el contacto con fluidos corporales como la sangre, secreciones orgánicas con sangre como pus, vómito, orina, y los provenientes de cavidades estériles como el líquido céfalo raquídeo $(6,11)$. Los accidentes corto-punzantes con material contaminado, el contacto con portadores de enfermedades infectocontagiosas y la falta de elementos de protección personal son realidades que los afectan diariamente (12).

Los largos turnos repercuten indudablemente en su vida, ya que no coinciden en las actividades sociales, resintiéndose la vida familiar y las redes de apoyo social $(12,13)$, por otro lado, el riesgo de accidentes de trayecto se incrementa con la somnolencia y el cansancio (14).

El género, como fenómeno social, ha marcado la evolución de la enfermería profesional bajo el alero del patriarcado, el machismo y el poder de parte del hombre hacia la mujer, existiendo desigualdad de salario, de responsabilidades y sobre todo de trabajo en el hogar (15).

El objetivo de esta revisión de la literatura es analizar las características del "trabajo" en enfermería inmersa en el mundo globalizado de la época contemporánea, de acuerdo a los resultados obtenidos en diferentes publicaciones que se refieren al tema.

\section{MATERIAL Y MÉTODO}

Se realizó una búsqueda bibliográfica de los estudios cuyos descriptores primarios fueran "trabajo", "enfermería", "salud laboral" o "rol de la enfermera" y secundarios "enfermeras clínicas" o "atención de enfermería”, ubicados fundamentalmente en el título del artículo y que correspondiera efectiva y exclusivamente a alguna área del trabajo que desempeñara la/el enfermera/o en diferentes servicios a nivel hospitalario y centros de salud extra-hospitalarios. Se incluyeron trabajos a texto completo tanto de carácter cuan- 
titativo como cualitativo en inglés, español y portugués. Se descartaron aquellos estudios que, a pesar de referirse al tema del trabajo en enfermería, se relacionaban básicamente con algunos síndromes o patologías como la fatiga o el Burnout, a excepción que presente una descripción importante de las condiciones en las cuales trabajan los profesionales de enfermería y la satisfacción que presentan respecto de éstas. La búsqueda se realizó en bases de datos informatizadas: Web of Science, MEDLINE, PubMed, SciELO, LILACS, BIREME y CUIDEN. La revisión proporcionó 39 estudios que cumplían los siguientes criterios de inclusión: a) Estudios cuantitativos y cualitativos sobre el trabajo realizado por enfermeras/os en los diferentes servicios; b) Escritos en español, inglés o portugués; c) Investigaciones publicadas desde el año 2000 al 2010; d) Realizadas en Centros Asistenciales.

\section{RESULTADOS}

Se pueden distinguir las siguientes temáticas abordadas en las investigaciones:

\section{Actividades realizadas por las/os enferme- ras/os (17 artículos)}

En el área asistencial las actividades se relacionan con: valoración física, remisión y control de síntomas, trabajo de rescate, admisión de los usuarios al servicio, triage, control de signos vitales, administración de tratamiento, cuidados relacionados con la higiene y alimentación, orientación a los familiares sobre el estado de salud de los pacientes y preparado de materiales en centrales de esterilización para las actividades de los demás servicios, entre otras (16-19). Actividades administrativas y burocráticas como el seguimiento de exámenes, traducción de la letra en las indicaciones, coordinar y supervisar actividades desarrolladas por los agentes comunitarios de salud, reuniones de coordinación, mantenimiento de la atención, pedidos, compras, control de la medicación, gestión del transporte y locomoción del paciente, contestar el teléfono, control de la presencia del trabajador, distribución de la tarea en caso de ausencia y la exigencia de productividad, entre otras (16-26). Las actividades administrativas o de gestión son las que demandan mayor tiempo, por lo cual el confort y tratamiento directo, en reiteradas ocasiones, son delegados al personal paramédico (17).

En general las labores de enfermería implican estar al tanto y pendiente de todo, absorbiendo las necesidades asistenciales y las de los demás participantes del equipo de salud (27), contribuyendo además a los planes estratégicos de la institución (28).

En el área de salud ocupacional desarrolla actividades de educación, promoción de la salud y prevención de los accidentes del trabajo y enfermedades profesionales junto con la asistencia directa a los trabajadores (29).

Las/os enfermeras/os que se desempeñan en el área gerencial describen como las actividades mas importantes la gestión con las personas, procesos y centrada en el usuario (30), lo que significa proveer guías para el equipo de enfermería, supervisar el cuidado de enfermería, manejo financiero, planeación, evaluación y organización de la carga de trabajo de acuerdo al staff y las condiciones de trabajo, el flujo de pacientes, las actividades rutinarias de los servicios, realizar reuniones de equipo y actividades de cooperación, encarando de manera responsable las actividades comunicacionales y el manejo de información $(31,32)$.

\section{Condiciones de trabajo en las cuales se des- empeña (20 artículos)}

La realidad económica mundial muestra cambios político-sociales que han llevado a la adaptación de los puestos de trabajo, disminuyendo la calidad de las condiciones, bajas remuneraciones y la creación de siste- 
mas que permiten la figura de prestadores de servicios, agentes externos que agotan la estabilidad laboral ofreciendo ingresos de acuerdo a las horas trabajadas, lo que, debido a la saturación del mercado, se traduce en cesantía $(16,29,33-37)$. El ambiente organizacional clínico es impositivo, inflexible, falto de reconocimiento y lleno de normas incuestionables, donde la competencia es una característica que menoscaba las relaciones establecidas entre las/os colegas, llevando a la desvalorización, la desmotivación del grupo de trabajo y desintegración profesional. Por otro lado, doblan su jornada de trabajo manifestando falta de tiempo para descansar, organizar y aprender $(23,38,39)$.

El trabajo implica riesgos físicos derivados del levantamiento y manipulación de peso, posición de trabajo de pie por largos periodos de tiempo y la alternancia de turnos. Riesgos biológicos, evidenciadas en el contacto con pacientes que presentan enfermedades infecto-contagiosas o la manipulación de materiales contaminados, y riesgos psicológicos derivados del trabajo en turnos, la subordinación, la descalificación, el trabajo fragmentado, la repetición de las tareas y el género $(29,34,35)$. El ritmo de trabajo es acelerado debido a la constante exigencia de no cometer errores que impliquen omisiones, negligencias o información deficiente (30). La desorganización institucional se evidencia en la falta de condiciones óptimas, falta de materiales, recursos humanos escasos y personal no entrenado, lo que trae como consecuencia la improvisación $(30,40)$, sumado a ello, malas relaciones interpersonales, comunicación deficiente y escaso trabajo en equipo entre las/os enfermeras/os y los médicos, lo que provoca alto desgaste y pobre calidad de atención a los pacientes (41).

Bajo reconocimiento social, debido a que los usuarios del sistema no distinguen las funciones específicas que desempeña en relación a otros profesionales o técnicos, esto provoca confusión que se traduce en un mimetismo social, causando frustración e incertidumbre referente al impacto del trabajo realizado (42).

A pesar de todo lo mencionado, se describen características que contribuyen a optimizar las condiciones de trabajo como el compromiso, el acceso a la información, el apoyo, la posibilidad de recursos y oportunidades, teniendo un impacto positivo en el clima de seguridad y grado de cohesión de estos profesionales con los médicos y directivos, y en última instancia, en el estrés experimentado durante las actividades laborales $(43,44)$.

\section{Género (4 artículos)}

Enfermería, al ser una profesión con una denominación intrínsecamente femenina, presenta la carga del constructo patriarcal que ha permanecido en la historia de la humanidad, debiendo compatibilizar la vida laboral y familiar, ya que al llegar al hogar extiende su función de gestión del cuidado con sus hijos, la casa y su pareja $(39,45,46)$. Por otro lado, la enfermera especialista en geriatría prolonga esta actividad a aquellos familiares de avanzada edad (47).

\section{Consecuencias del trabajo para las/os en- fermeras/os (6 artículos)}

Los profesionales, al estar expuestos a riesgos laborales, presentan daños en la salud física y psicológica, expresándose en enfermedades músculo-esqueléticas, trastornos gastrointestinales, sentimientos de impotencia, frustración, tristeza, problemas de sueño, dificultad en las percepciones, ansiedad, cansancio, fatiga, irritabilidad, falta de concentración, depresión, pesimismo, falta de comunicación, baja productividad, falta de creatividad y Síndrome de Burnout (34, 38, 48-50). El trabajo en turnos genera consecuencias que van más allá de lo laboral; los riesgos de las 
actividades extenuantes en este sistema se han manifestado frecuentemente cuando las enfermeras retornan a sus hogares, especialmente aquellas que conducen, pues han reportado momentos de somnolencia, poniendo en riesgo sus vidas y las de los transeúntes (14).

\section{Satisfacción laboral (8 artículos)}

Las/os enfermeras/os y las personas que presentan algún grado de dependencia y que demandan ayuda para sí y para sus familias, logran una estrecha comunicación basada en respeto y comprensión $(43,51)$. El trabajar en equipo y alcanzar las expectativas generadas al realizar tareas específicas en el lugar de trabajo y ser técnicamente hábil en los cuidados, reportando reconocimiento al profesional, producen satisfacción $(51,52)$.

El trabajo es considerado como una oportunidad de crecimiento personal, que al ser reconocido genera satisfacción a pesar de la presencia de estrés (52).

En contraposición, la insatisfacción en el trabajo surge cuando las/os enfermeras/os dedican mayor parte del tiempo en realizar actividades administrativas que no les permiten atender directamente a los pacientes a cabalidad (4) o cuando no existe el debido reconocimiento de parte de los superiores o la comunidad, los derechos de los trabajadores se pasan a llevar o no son tomados en cuenta, las remuneraciones no son justas y la relaciones jerárquicas e interpersonales son conflictivas $(40,53)$.

El liderazgo, el clima laboral creativo, un mayor compromiso en el cuidado y una mayor autonomía, son factores estrechamente correlacionados con la satisfacción laboral, adquiriendo mayor preponderancia la autonomía. Por otra parte, si se asocia la satisfacción laboral con la satisfacción con la carrera desarrollada, son predictores de la satisfacción en la vida $(54,55)$.

\section{DISCUSIÓN Y CONCLUSIÓN}

A través de la evidencia se identifica una alta demanda psicológica y física hacia el rol de enfermería, especialmente en unidades de cuidado crítico debido a diferentes factores, uno de ellos se establece en la estrecha relación con el paciente y la familia, creando un lazo de dependencia con alto compromiso en pos de otorgar cuidado de calidad. Las mayores demandas de trabajo se relacionan con: vivenciar el sufrimiento del paciente, tomar decisiones bajo presión, proporcionar cuidado a familias con requerimientos emocionales, falta de tiempo para dar soporte emocional al paciente por actividades administrativas, orientación familiar, carga horaria semanal y turnos de trabajo $(56,57)$. Esto se ha evaluado preferentemente en las unidades de cuidados críticos, ya que las características del paciente, gravedad de la enfermedad, la supervivencia y el tiempo de permanencia de éstos en estas unidades, demanda mayor cantidad de horas de dedicación de parte del/la enfermero/a $(5,58)$.

Por otra parte, las/os enfermeras/os se enfrentan diariamente a factores de riesgo psicosociales asociados a las características propias de la organización, como la falta de reconocimiento, la desigualdad y la sobrecarga de trabajo. Además, actúa como intermediario entre el paciente, la familia y el médico, por lo tanto, está en continua presión de trabajo (59).

En cuanto al género en enfermería, se evidencia que la mayoría de las enfermeras, terminando su jornada laboral, continúan con las labores en el hogar, sumado a ello, el presentar uno o más hijos aumenta la sobrecarga de trabajo; desde este punto de vista, está obligada a conciliar estos dos espacios, lo doméstico y lo público, bajo condiciones de menor remuneración en relación a los hombres y con una carga de trabajo excesiva (46). 
En la atención primaria se muestra insatisfacción importante de parte de los trabajadores en temas como la relación con los jefes, con los compañeros, en los horarios y la percepción de bajo control en la tarea debido a la disminución de la oportunidad en la participación de la toma de decisiones, lo que repercute además en el trato que se le da al paciente $(60)$.

Se podría concluir que juegan un papel relevante para el desarrollo del trabajo en enfermería las condiciones en que se desenvuelve lo laboral. Estas condiciones constantemente tienen necesidad de adaptación e improvisación de materiales y equipos, lo que provoca consecuencias importantes en los trabajadores, con signos de miedo, ansiedad, estrés, enojo, dolor de piernas, espalda, cefalea y fatiga (61). Por otro lado, se ha evidenciado que las enfermeras que realizan turnos, que implica trabajo nocturno en instituciones hospitalarias, se ven muy perjudicadas, presentando síntomas de embotamiento y trastornos del sueño (62).

La carga de trabajo ha sido evaluada en diferentes servicios $(3,5,63)$. Debido a que esta profesión comprende la experiencia del cuidado enfrentando al ser humano en forma holística, debe desempeñarse con autonomía para realizar actividades de protección, promoción de la salud, prevención y recuperación de la enfermedad. En este sentido la/el enfermera/o, para entregar el cuidado, debe tener una amplia visión de los procesos de vida, enfrentando sentimientos complejos con una carga física y psíquica importante (4). La carga mental de trabajo (CMT) se asocia significativamente al tipo de servicio, al nivel profesional y a la duración de la jornada laboral. Por otro lado, se ha encontrado menor CMT en los servicios de hemodiálisis y unidad de cuidados intensivos (UCI) pediátricos, contrariamente a los servicios de urgencias extrahospitalarias, servicios de urgencias generales, reanimación, urgencias pediátricas y UCI adulto (64).

El estrés y el síndrome de Burnout son te- máticas reiteradas en el trabajo de enfermería como consecuencias de las labores extenuantes y los sistemas de turnos; en este sentido existen estudios que evidencian que el estrés es un predictor importante del síndrome de Burnout y que las demandas en el trabajo son los determinantes más importantes en el desgaste emocional, mientras que los recursos que la persona presenta para enfrentarlo predicen la despersonalización. El agotamiento y despersonalización se vinculan a problemas psicosomáticos que junto al compromiso profesional se asocian a la intención de abandonar la profesión de enfermería (65). Las enfermeras, entre el personal de salud, son las que corren mayor riesgo de sufrir este síndrome, especialmente las enfermeras oncológicas o las que trabajan con pacientes con SIDA (66).

El profesional de enfermería presenta la gran responsabilidad de ser líder para trabajar por un objetivo institucional común, visualizando claramente las problemáticas de los servicios con competencia técnica y humana, donde juega un papel preponderante la construcción colectiva del cuidado al articular ampliamente las actividades con el resto del equipo de salud (67).

Los roles que debe cumplir la enfermera en el ambiente laboral y en la vida le provocan desgaste, frustración y síndromes patológicos físicos y psíquicos, ya que son múltiples y simultáneos, lo que da a entender que es necesario evaluar los lugares de trabajo para mejorar los procesos, la infraestructura y la relación del recurso con la demanda de atención. Al trabajador que imparte el cuidado se le ha desatendido y no se ha tomado en cuenta que también forma parte de un entorno social-familiar que es igual o más importante que lo laboral. La eficiencia y eficacia de los servicios dependen de la satisfacción, bienestar y calidad de vida de los trabajadores, por lo tanto, la institución debe invertir en recursos para mantener óptimas condiciones laborales; de ello depende, en este caso en particular, la calidad de la atención brindada a los usuarios 
de los sistemas de salud y el prestigio de la empresa. En esto, la investigación continua y la publicación de resultados que permitan mostrar evidencias suficientes para el cambio de paradigma en el ámbito laboral de estos profesionales son herramientas fundamentales. Por otro lado, no solo la empresa debe asumir la responsabilidad, los trabajadores de enfermería deben organizarse para exigir sus derechos, a la vez, utilizar su habilidades y herramientas para mejorar los ambientes laborales mediante una actitud crítica, pero positiva, frente al trabajo y la manera de realizar las actividades, promoviendo la mejora continua de la gestión del cuidado.

\section{REFERENCIAS}

1. Jiménez A, Acevedo D, Salgado L, Moyano E. Cultura trabalho-família e compromisso organizacional numa empresa de serviços. Psicol Estud. 2010; 14(4): 72938.

2. Ferreira E, Prado M, Prado C, Pavan P, Fernandes G, Barrionuevo B. Prazer e sofrimento no processo de trabalho do enfermeiro docente. Rev Esc Enferm USP 2009; 43(esp 2): 1292-6.

3. Panunto M, Guirardello E. Nursing workload at a gastroenterology unit. Rev Lat Am Enfermagem. 2009; 17(6): 1009-14.

4. Magela G, Salles M, Cunha V. Sentimentos vivenciados pelos profissionais de enfermagem que atuam em unidade de emergência. Rev Bras Enferm. 2010; 62(6): 856-62.

5. Andrade L, Grillo K. Fatores associados à carga de trabalho de enfermagem em unidade de terapia intensiva. Rev Esc Enferm USP. 2007; 41(4): 645-52.

6. Valenzuela S, Sanhueza O, Riquelme N, Fernández M, Núñez R. Salud laboral: situación de los accidentes de trabajo en el equipo de enfermería. Enferm Clin. 2002; 13(2): 94-102.
7. Murofuse N, Abranches S, Napoleao A. Reflections on stress and burnout and their relationship with nursing. Rev Lat Am Enfermagem. 2005; 13(2): 255-61.

8. Astudillo P, Alarcón AM, Lema M. Protectores de estrés laboral: Percepción del personal de enfermería y médicos, Temuco, Chile. Cienc. enferm. 2009; XV(3): 111-22.

9. Escribá-Aguir V, Burguete-Ramos D. ¿Cómo perciben las enfermeras de transplantes las causas del estrés laboral? Index Enferm. 2005; 14(50): 15-9.

10. Van Bogaert P. Commentary on Kowalski C, Ommen O, Driller E, Ernstmann N, Wirtz M, Kohler T, et al. (2010). Burnout in nurses-the relationship between social capital in hospitals and emotional exhaustion. J Clin Nurs 19, 1654-1663. J Clin Nurs. 2011; 20(5-6): 913-4.

11. Velásquez G. Prevención del riesgo biológico en los trabajadores de la salud. Cuaderno ASCOFAME. 1999; 40(5): 2-9.

12. Barcellos R, Cruz M, Almeida L. Riscos ocupacionais e alterações de saúde entre trabalhadores de enfermagem brasileiros de unidades de urgência e emergência. Cienc enferm. 2010; XVI(2): 69-81.

13. Retamales O, Behn V, Merino J. Funcionamiento familiar de trabajadores-jefes de hogar en sistema de turnos de una empresa de Talcahuano, Chile, año 2003. Cienc. enferm. 2004; X(1): 23-30.

14. Scott L, Hwang W, Rogers A, Nysse T, Dean G, Dinges D. The relationship between nurse work schedules, sleep duration, and drowsy driving. Sleep 2007; 30(12): 1801-7.

15. Bonino L. Micromachismos, el poder masculino en la pareja "moderna". En: Espada Ch, editor. Voces de Hombres por la Igualdad. España: Armería; 2008. p. 89109.

16. De Mattia A, Andres V. A saúde do trabalhador de enfermagem sob a ótica da gerência. Rev Bras Enferm. 2004; 57(4): 453-8. 
17. Bernardo M. Ações de enfermagem no cenário do cotidiano de uma instituição psiquiátrica. Rev Lat Am Enfermagem. 2003; 11(5): 678-84.

18. Cardoso A. O trabalho da enfermagem: análise e perspectiva. Rev Bras Enferm. 2003; 56(6): 669-673.

19. Ratner D, Correa F. O trabalho do enfermeiro nos equipamentos de saúde mental da rede pública de Campinas-SP. Rev Lat Am Enfermagem. 2001; 9(5): 77-82.

20. Schimith M, Dias M. O enfermeiro na equipe de saúde da família: estudo de caso. Rev. enferm. UERJ. 2009; 17(2): 252-6.

21. Vilela S, Moraes M. A prática de enfermagem em serviços abertos de saúde mental. Rev. enferm UERJ. 2008; 16(4): 501-6.

22. Medeiros A, Barros S. O trabalho de enfermagem no hospital dia na perspectiva da reforma psiquiátrica em João Pessoa-Paraíba. Rev Esc Enferm USP. 2005; 39(3): 310-6.

23. Monteiro B, Furtado F, Braga F, Soares C. Jornada de trabalho: fator que interfere na qualidade da assistência de enfermagem. Texto contexto-enferm. 2006; 15(3): 442-8.

24. Leary A, Crouch H, Lezard A, Rawcliffe C, Boden L, Richardson A. Dimensions of clinical nurse specialist work in the uk. Nurs Stand. 2008; 23(15-17): 40-4.

25. Mackinnon K. Labouring to nurse: the work of rural nurses who provide maternity care. Rural Remote Health. 2008; 8(4): 1047.

26. Silva A, Peduzzi M. O trabalho de enfermagem em laboratórios de análises clínicas. Rev Lat Am Enfermagem. 2005; 13(1): 65-71.

27. Guarín G, Castrillón M. El mundo de la enfermera: "uno aquí no se sienta". Contexto de los cuidados. Invest Educ Enferm. 2009; 27(2): 226-34.

28. Gindri G, Freditas H, Zamberlan C, Santini R. Apercepção dos profissionais da equipe de enfermagem sobre o trabalho dos enfermeiros. Cogitare Enferm. 2005; 10(1): 34-41.

29. Pinho E, Da Costa N, Lúcia A. A saúde do trabalhador na concepção de acadêmicos de enfermagem. Rev Esc Enferm USP. 2007; 41(3): 355-62.

30. Ribeiro A, Palucci M. Relação tempo-violência no trabalho de enfermagem em emergência e urgência. Rev Bras Enferm 2006; 59(3): 337-43.

31. Surakka T. The nurse manager's work in the hospital environment during the 1990s and 2000s: responsibility, accountability and expertise in nursing leadership. J Nurs Manag. 2008; 16(5): 525-34.

32. Gardner G, Gardner A, Middleton S, Della P, Kain V, Doubrovsky A. The work of nurse practitioners. J Adv Nurs. 2010; 66(10): 2160-9.

33. Dal Pai D, Lautert L. Work under urgency and emergency and its relation with the health of nursing professionals. Rev Lat Am Enfermagem. 2008; 16(3): 439-44.

34. Batista J, Casquel C, Aparecido J. Retraining due to illness ant its implications in nursing management. Rev Lat Am Enfermagem. 2010; 18(1): 87-93.

35. Ferreira D, Silva A, Lúcia M, Barbosa M. Ser trabalhador de enfermagem da unidade de centro de material: uma abordagem fenomenológica. Rev Esc Enferm USP. 2007; 41(4): 675-82.

36. Clarke Sp. Hospital work environments, nurse characteristics, and sharps injuries. Am J Infect Control. 2007; 35(5): 302-9.

37. Gutiérrez M, Hernández J, Villanueva A. Significado que tienen las condiciones laborales actuales para la calidad de vida como factor de desarrollo humano de la enfermera(o). Hacia la promoción de la salud. 2004; 9(119): 131.

38. Aparecida M, Lúcia V. A relação entre o trabalho, a saúde e as condições de vida: negatividade e positividade no trabalho das profissionais de enfermagem de um hospital escola. Rev Lat Am Enfermagem. 2006; 14(4): 517-25. 
39. Baggio M, Formaggio F. Trabalho, Cotidiano e o profissional de enfermagem: o significado do descuidado de si. Cogitare Enferm. 2012; 13(1): 67-74.

40. da Silva R, Pereira J. Imagens e representações da enfermagem acerca do stress e sua influência na atividade laboral. Rev Esc Enferm USP. 2010; 44(3): 694-701.

41. Kanai-Pak M, Aiken Lh, Sloane Dm, Poghosyan L. Poor work environments and nurse inexperience are associated with burnout, job dissatisfaction and quality deficits in japanese hospitals. J Clin Nurs. 2008; 17(24): 3324-9.

42. Hiromi L, Januário M. Processo de trabalho do enfermeiro: visão de professores de uma universidade pública. Acta paul. enferm. 2008; 21(3): 481-6.

43. Mark B, Hughes L, Belyea M, Chang Y, Hofmann D, Jones C, et al. Does safety climate moderate the influence of staffing adequacy and work conditions on nurse injuries? J Safety Res. 2007; 38(4): 431-46.

44. Almost J, Laschinger H. Workplace empowerment, collaborative work relationships, and job strain in nurse practitioners. J Am Acad Nurse Pract. 2002; 14(9): 40820.

45. Spíndola T, Da Silva R. Trabalho versus vida em família. Conflito e culpa no cotidiano das trabalhadoras de enfermagem. Cienc. enferm. 2004; X(2): 43-52.

46. Báez G, Castro C, Ramírez A, Estrada J. Determinación de fatiga física en enfermeras que laboran en el área de emergencia del IMSS. Memorias del VII Congreso Internacional de Ergonomía. 3 al 5 de noviembre de 2005. México. México: Sociedad de Ergonomistas de México. p. 54-63.

47. Gattuso S, Bevan C. Mother, daughter, patient, nurse: women's emotion work in aged care. J Adv Nurs. 2000; 31(4): 892-9.

48. Zacché L, Iglesias A, Fernandes P. Sofrimento psíquico em trabalhadores de enfermagem de uma unidade de oncologia. Psicol Estud. 2007; 12(3): 475-81.

49. Magela G, Cunha V, Reis G. The nursing professional in an intensive therapy unit. Acta paul. enferm. 2008; 21(2): 294-9.

50. Hanrahan N, Aiken L, McClaine L, Hanlon A. Relationship between psychiatric nurse work environments and nurse burnout in acute care general hospitals. Issues Ment Health Nurs 2010; 31(3): 198-207.

51. Christiansen B. Good work-how is it recognised by the nurse? J Clin Nurs 2008; 17(12): 1645-51.

52. Trevisan J, Do Carmo M, Garanhani M. Sentimentos de prazer entre enfermeiros de unidades de terapia intensiva. Cienc. enferm. 2009; XV(3): 45-53.

53. Karlsson I, Ekman S1, Fagerberg I. A difficult mission to work as a nurse in a residential care home-some registered nurses' experiences of their work situation. Scand J Caring Sci. 2009; 23(2): 265-73.

54. Sellgren S, Ekvall G, Tomson G. Leadership behaviour of nurse managers in relation to job satisfaction and work climate. J Nurs Manag. 2008; 16(5): 578-87.

55. Nemcek M, James G. Relationships among the nurse work environment, self-nurturance and life satisfaction. J Adv Nurs. 2007; 59(3): 240-7.

56. Baernholdt M, Mark B. The nurse work environment, job satisfaction and turnover rates in rural and urban nursing units. J Nurs Manag. 2009; 17(8): 9941001.

57. Costa L, De Brito E. Nurses' attention demands in the work setting. Rev Lat Am Enfermagem. 2010; 15(1): 27-33.

58. Pazetto A, Viski S, Yamaguchi I. Carga de trabalho de enfermagem e sua relação com a gravidade dos pacientes cirúrgicos em UTI. Acta paul. enferm. 2010; 19(1): 16-20.

59. Gómez M, Álamo M, Amador M, Ceacero F, Mayor A, Muñoz A, et al. Estudio del seguimiento del desgaste profesional en relación con factores organizativos en el personal de enfermería de medicina interna. Med Segur Trab. 2009; 55(215): 52-62. 
60. Scherlowski H, Chaves M, Gomes V, De Souza M, Henriques F. Organização do trabalho de enfermagem na atenção básica: uma questão para a saúde do trabalhador. Texto contexto-enferm. 2009; 18(2): 206-14.

61. De Oliveira N, Machado D, Lima E, Tavares C, Da Silva C, Costa M. Repercussões psicofísicas na saúde dos enfermeiros da adaptação e improvisação de materiais hospitalares. Esc Anna Nery. 2010; 14(2): 236-43.

62. Palucci M. Turnos alternantes: fadiga mental de enfermagem. Rev Lat Am Enfermagem. 1995; 3(1): 59-78.

63. Janzantte A, Viski S, Yamaguchi I. Carga de trabalho de enfermagem para quantificar proporção profissional de enferma- gem/paciente em UTI cardiológica. Rev Esc Enferm USP 2008; 42(4): 873-80.

64. González J, Moreno B, Garrosa E. Carga mental y fatiga en servicios espaciales de enfermería. Rev Latinoam Psicol. 2005; 37(3): 477-92.

65. Jourdain G, Chenevert D. Job demands-resources, burnout and intention to leave the nursing profession: a questionnaire survey. Int J Nurs Stud. 2010; 47(6): 709-22.

66. Bernardi M, Catania G, Marceca F. The world of nursing burnout. A literature review. Prof Inferm. 2005; 58(2): 75-9.

67. Stein D, Stein M, Macêdo F, Lorenzini A. O papel do enfermeiro no contexto hospitalar: a visão de profissionais de saúde. Cienc Cuid Saúde. 2008; 7(3): 319-26. 